\title{
Quantitative measurements of fuel concentrations in direct- injection methanol-n-hexane sprays by laser induced breakdown spectroscopy
}

\author{
Zhifei Zhang ${ }^{* 1}$, Tie Li ${ }^{\star 1}, 2$, Ning Wang1, Yijie Wei ${ }^{1}$ \\ 1 State Key Laboratory of Ocean Engineering, Shanghai Jiao Tong University, Shanghai, \\ China \\ ${ }^{2}$ Collaborative Innovation Center for Advanced Ship and Deep-Sea Exploration, Shanghai \\ Jiao Tong University, Shanghai, China \\ ${ }^{*}$ Corresponding author email: litie@sjtu.edu.cn
}

\begin{abstract}
Methanol is considered to be one of promising alternative fuels, and direct-injection (DI) of methanol-gasoline blends is attracting more and more interest due to the thermal efficiency advantages. However, quantitative measurements of component concentrations in DI methanol-gasoline blend sprays are still difficult. In this study, a novel approach based on the laser induced breakdown spectroscopy (LIBS) is developed to quantitatively and simultaneously measure the gas concentrations of $n$-hexane and methanol in methanolgasoline blend sprays in a constant volume vessel. Firstly, the calibrations between peak intensity ratios (PIR) of $\mathrm{H656/N745}$ and atomic number ratios (ANR) of $\mathrm{H} / \mathrm{N}$, and PIR of O777/N746 and ANR of O/N are established respectively for three kinds of fuels (M0, M50, M100). The results show a good consistency of calibration curves for different fuels and different ambient pressures, suggesting the potentials of LIBS to measure multi-component fuels even at varied-pressure conditions. Then, the high-speed shadowgraph and diffused back-illumination method are employed to simultaneously image the entire spray and the liquid phase, and therefore determine the LIBS measuring positions. Finally, the fuel concentration measurements in M0 and M15 sprays are conducted and the radial distribution and the effects of ambient pressure are investigated. It is found that the blending fraction in M15 spray is increased with the radial distance increasing due to the lower boiling temperature and higher vapor diffusivity of methanol. The equivalence ratios in both $\mathrm{M} 0$ and M15 sprays are reduced with the increased ambient pressure, which can be attributed to the larger air density while nearly constant entrainment speed at higher ambient pressure. These results are believed to be valuable for development of numerical models and design of DI combustion systems.
\end{abstract}

\section{Keywords}

Methanol-gasoline blends; Fuel concentration; LIBS; Quantitative measurements.

\section{Introduction}

Nowadays, higher efficiency and lower emissions are the main targets of spark ignition (SI) engines. To meet the strict requirements, sustainable renewable fuels are attracting more and more interest. Methanol is considered to be one of promising alternative fuels [1-3]. Adding methanol into gasoline has several advantages for direct-injection spark ignition (DISI) engines. Firstly, due to the high latent heat of evaporation, direction injection (DI) of methanol can enhance the charge cooling effect and therefore reduce the risk of knock [4]. In addition, the methanol has a higher octane number than gasoline. Therefore, the engines can operate at a higher compression ratio, improving the thermal efficiency. Moreover, the soot formation can be reduced because of the oxygen content in methanol [5]. However, it is notable that adding methanol to gasoline lead to the worse atomization and evaporation characteristic 
because of the higher boiling temperature and the larger latent heat of evaporation. The worse atomization and evaporation might lead to fuel impingement on cylinder surface, incomplete droplet evaporation and heterogeneous fuel-air mixture, causing higher PM emissions [6]. As a consequence, investigations on spray evaporation and fuel-air mixing process is of great significance for DISI engines consuming methanol-gasoline blends.

In DI methanol-gasoline blend sprays, due to the difference in evaporation and diffusion characteristics, the methanol and gasoline might evaporate and mix with air in different time and space, leading to an inhomogeneous vapour distributions of two fuels [7, 8]. Moreover, the ignition characteristics and flammable range of methanol and gasoline are distinct. Therefore, simultaneous measurements of concentration distributions of methanol and gasoline is necessary since they directly determine the following ignition and combustion process. In addition, the fuel concentration information is necessary for understanding fuel-air mixing mechanism and calibration of spray model. To achieve the quantitative concentration measurements, laser-based techniques are commonly used, such as the planar laser induced fluorescence (PLIF) [9, 10], the laser absorption and scattering (LAS) [11, 12], the laser induced breakdown spectroscopy (LIBS) $[13,14]$ etc. The PLIF technique is a most popular technique for concentration measurements. However, for a number of fluorescent substance, quenching could be enhanced by oxygen, making it nonlinear between the fluorescence intensity and the targeted molecular concentration [15]. As a result, the accurate quantification by PLIF would be difficult for methanol-based fuels. Until now, only few groups have attempted to employ the PLIF technique to qualitatively characterize the distributions of methanol or ethanol-based fuels $[16,17]$. Moreover, in the PLIF measurements with tracer doped into fuels, it requires that the tracer should have excellent traceability with the targeted fuels. Differences in the physical properties such as molecular mass and diffusion coefficient between the tracers and fuels could introduce measurement errors. In the LAS technique, the test fuel is required to strongly absorb the light with certain wavelength but its fuel vapor is almost transparent at the other wavelength [18]. In addition, the fuel sprays are assumed to be symmetric, which could degrade the measurement accuracy.

LIBS is an atomic emission spectroscopic technique in which a high-energy laser pulse is tightly focused on the target and the resulting plasma emission is dispersed and analyzed. Compared with other laser-based techniques, LIBS has some obvious benefits such as the strong signals, superiority in quantification, capability of multi-element simultaneous analysis and applicability to all sample types like solids, liquids and gases [19-21]. More importantly, LIBS is based on excitations determined by elemental concentration rather than molecular structure, therefore it enables the concentration measurements of multi-component fuels and oxygenated fuels.

In summary, although the fuel concentration information in DI methanol-gasoline blend sprays is critical, the quantitative measurement is still a difficulty and the studies are very limited. In this study, a novel approach based on LIBS has been proposed to simultaneously and quantitatively measure $n$-hexane and methanol concentrations in DI methanol-n-hexane blend sprays by using multiple atomic lines.

\section{Experimental apparatus and procedure}

Figure 1 shows the schematic diagram of the LIBS measurement system. The methanolgasoline spray injections were performed in an optically-accessible constant volume vessel (CVV) under varied ambient and injection conditions. On the top of the CVV was installed a single-hole injector with the hole diameter of $0.18 \mathrm{~mm}$. The mixture temperature in the CVV could be varied from room temperature of $300 \mathrm{~K}$ to $470 \mathrm{~K}$ by using an electric heater from 


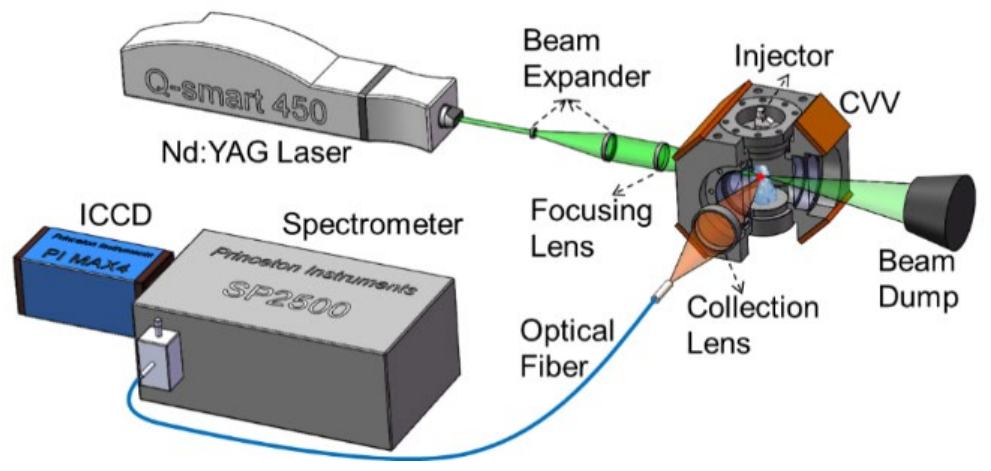

Figure 1. Schematic diagram of the LIBS measurement system.

Table 1 - Experimental specifications.

\begin{tabular}{ll}
\hline Ambient gas & $\mathrm{N}_{2}$ \\
Ambient density $\left(\mathrm{kg} / \mathrm{m}^{3}\right)$ & 15 \\
Ambient pressure $(\mathrm{bar})$ & 20 \\
Ambient temperature $(\mathrm{K})$ & 460 \\
Nozzle hole diameter $(\mathrm{mm})$ & 0.18 \\
Injection pressure $(\mathrm{MPa})$ & 60 \\
Injection duration $(\mathrm{ms})$ & 1.5 \\
\hline
\end{tabular}

\begin{tabular}{lll}
\multicolumn{3}{c}{ Table 2 - Fuel properties. } \\
\hline Fuel & N-hexane & Methanol \\
\hline Molecular weight & 86 & 32 \\
Oxygen content $(\%)$ & 0 & 50 \\
Boiling point $(\mathrm{K})$ & 341.7 & 337.5 \\
Density $\left(\mathrm{kg} / \mathrm{m}^{3}\right)$ & 678 & 792 \\
Lower heat value $(\mathrm{MJ} / \mathrm{kg})$ & 44.8 & 19.9 \\
Enthalpy of vaporization $(\mathrm{kJ} / \mathrm{kg})$ & 384 & 1103 \\
Kinematic viscosity $\left(\mathrm{mm}^{2} / \mathrm{s}\right)$ & 0.57 & 1.00 \\
\hline
\end{tabular}

room temperature of $300 \mathrm{~K}$ to $470 \mathrm{~K}$ by using an electric heater and a temperature controller. A motor-driven mixing fan was employed to enable the fuel-air mixture as homogeneous as possible in the LIBS calibration process. During the test, the temperature of ambinet gas was controlled $460 \mathrm{~K}$. The injection duration $(\tau)$ was set to $1.5 \mathrm{~ms}$ during all measurements. The experimental conditions are summarized in Table 1. In the measurements, n-hexane was employed as the substitution of the gasoline. A certain proportion of methanol was added to $\mathrm{n}$-hexane to form the methanol-n-hexane blends. The fuel properties of methanol and nhexane are shown in Table 2.

The laser beam with 6.5-mm diameter from a 532-nm Nd:YAG laser (Quantel Q-smart 450) was firstly expanded to $50 \mathrm{~mm}$ in diameter and then focused into the CVV using a 200-mm focal length plano-convex lens. The pulse energy $(E)$ before the focusing lens was about 137 $\mathrm{mJ}$ with the standard deviation of $2.9 \%$. The plasma emission was collected by a $100-\mathrm{mm}$ focal length lenticular lens and then coupled into an optical bundle. The plasma signal was dispersed by a spectrometer (Princeton Instruments SP2500) with a 150-grooves/mm diffraction grating and finally imaged on an intensified charge coupled device (ICCD) camera (Princeton Instruments PI MAX4).

In this study, the LIBS measurements of fuel concentrations were conducted in vapor phase region of the methanol-gasoline blend sprays. Hence, the schlieren imaging technique and the diffused back-illumination (DBI) technique were simultaneously employed to determine the liquid and vapor phases in methanol-gasoline blend sprays. Two light beams from LED lamps in schlieren imaging and DBI systems were arranged in the orthogonal directions. Two high- 
speed cameras (Phantom V2012 and NAC MEMRECAM HX-6) were employed to capture the schlieren and DBI signals, respectively.

\section{Results and Discussion}

Figures 2(a-c) show the schlieren and DBI images of methanol-n-hexane blend sprays and the LIBS measuring positions at spray axial and radial directions. The employed fuel was the n-hexane blended with $15 \%$ methanol by volume (M15). The injection pressure and ambient pressure were $60 \mathrm{Mpa}$ and 20 bar, respectively. The images were acquired at $1.5 \mathrm{~ms}$ after the start of injection (ASOI). The schlieren image in Fig. 2(a) shows both the liquid and vapor phases of spray, and the DBI image in Fig. 2(b) exhibits the liquid phase of spray. The DBI image was firstly subtracted by the background image and then was binarized to obtain the liquid phase area. The threshold was determined by $10 \%$ of the maximum pixel intensity in the image. Then, the spray profile was detected by the edge detection algorithm. The tip penetration is defined as the distance from the nozzle hole exit to the spray front edge. The vapor penetration and the liquid penetration are expressed as $S_{V}$ and $S_{L}$, respectively. In Fig. 2(c), the red line represents the edge of liquid phase. The green points represent the LIBS measuring positions in the vapour phase of spray. Figure 2(d) shows the time evolutions of the vapor and the liquid penetrations. After the start of injection, the spray rapidly penetrates, however, the penetration speed becomes slower and slower due to the droplet breakup and the air resistance. At about $0.5 \mathrm{~ms}$ ASOI, the liquid and vapor penetrations are separated, indicating that the downstream spray is in the gas phase. After that, the liquid phase penetration generally keeps stable at $28 \mathrm{~mm}$ in spite of slight fluctuations. The measuring positions are selected at $40 \mathrm{~mm}$ from nozzle hole.

LIBS calibrations were conducted in CVV by established the homogeneous gas mixture of methanol-n-hexane blends and nitrogen. The laser induced breakdowns were achieved and corresponding emission spectra were collected. The spectral region between $630 \mathrm{~nm}$ and 790 $\mathrm{nm}$ was analyzed. Within $630-790 \mathrm{~nm}$, the atomic lines of hydrogen at $656 \mathrm{~nm}$, nitrogen at 742-746 $\mathrm{nm}$ and oxygen at $777 \mathrm{~nm}$ can be observed. All of the hydrogen, nitrogen and oxygen lines were employed to By using appropriate background and baseline subtraction method [13], the intensities of atomic lines can be accurately quantified. Then the correlations between line intensity ratios and atom concentration ratios were built up. Figure 3 shows the calibration results. The ambient pressure and temperature are 30 bar and $460 \mathrm{~K}$, respectively.

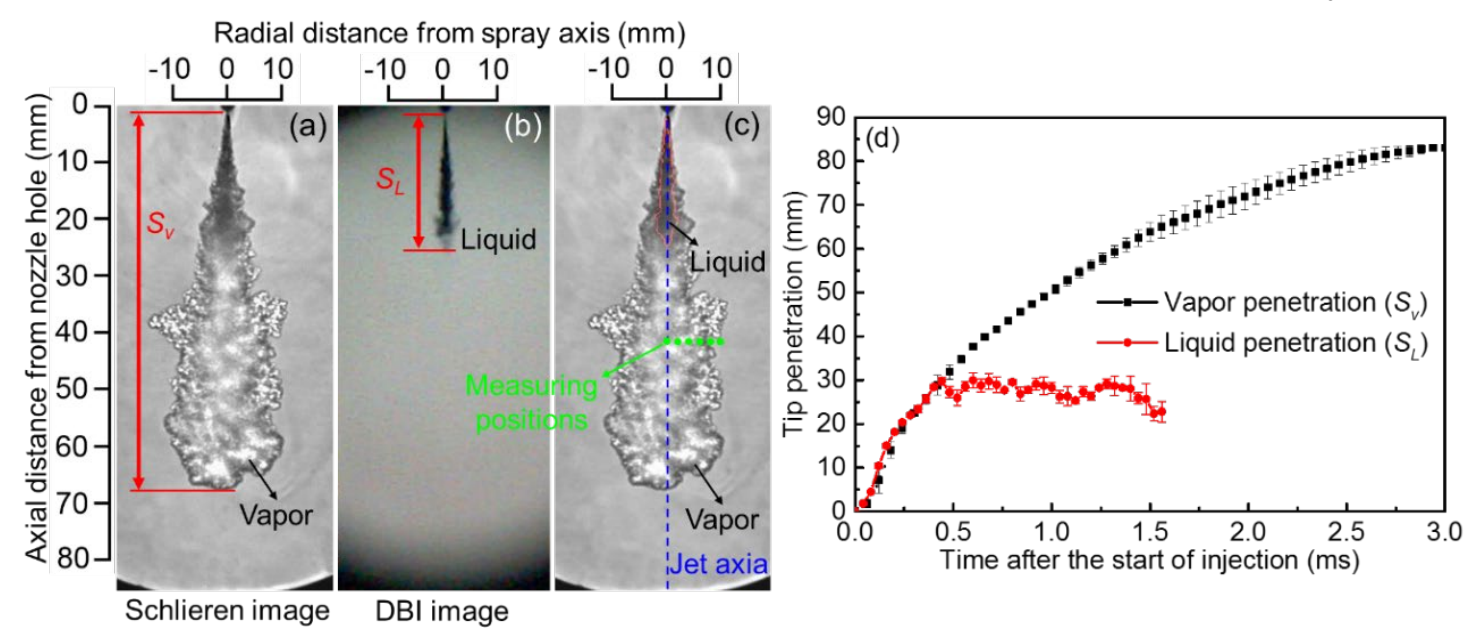

Figure 2. (a) The schlieren image and (b) DBI image of methanol-n-hexane blend sprays, (c) the LIBS measuring positions at spray axial and radial directions $\left(P_{i n j}=60 \mathrm{Mpa}, P_{a}=20 \mathrm{bar}, T_{a}=460 \mathrm{~K}, t_{A S O I}=1.5 \mathrm{~ms}, \tau=1.5 \mathrm{~ms}\right)$,

(d) the time evolutions of spray tip penetration and liquid phase tip penetration. 

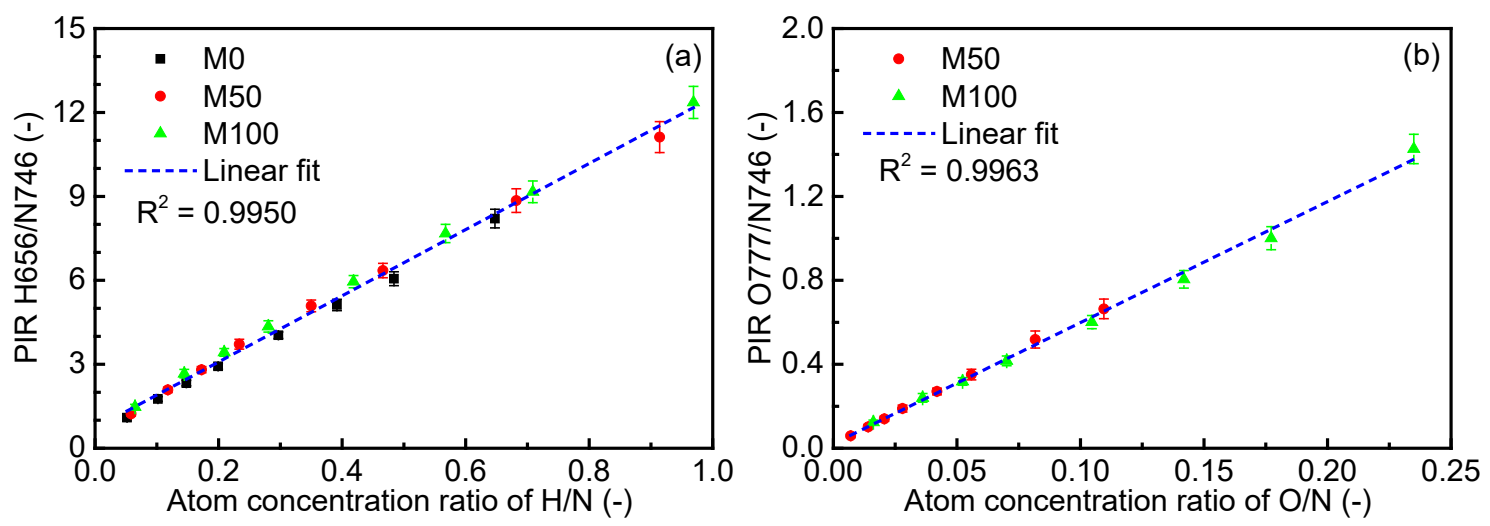

Figure 3. The calibration curves between (a) PIR of H656/N746 and atom concentration ratio of H/N and (b) PIR of $0777 / N 746$ and atom concentration ratio of $\mathrm{O} / \mathrm{N}$ at the ambient pressure of 30 bar and ambient temperature of $460 \mathrm{~K}\left(E=137 \mathrm{~mJ}, P_{a}=30 \mathrm{bar}, T_{a}=460 \mathrm{~K}\right)$.

Two PIRs (H656/N746 and O777/N746) were employed to determine the concentrations of three atoms (C, $\mathrm{O}$ and $\mathrm{N}$ ). The calibration curves between PIR of H656/N746 and atom concentration ratio of $\mathrm{H} / \mathrm{N}$ and calibration curves between PIR of 0777/N746 and atom concentration ratio of $\mathrm{O} / \mathrm{N}$ are depicted in Figs. 3(a) and (b), respectively. The results are the average values and the error bars represent the standard deviations for 100 single tests. Three fuels were tested. M0 and M100 denotes the pure n-hexane and pure methanol, and M15 means that the $n$-hexane contains $15 \%$ methanol by volume. It can be seen that the PIRs of $\mathrm{H} 656 / \mathrm{N} 746$ and $0777 / \mathrm{N} 746$ linearly correlate well with the atom concentration ratios of $\mathrm{H} / \mathrm{N}$ and $\mathrm{O} / \mathrm{N}$, respectively. The goodness of fit $\left(R^{2}\right)$ are larger than 0.99 , showing high measurement accuracies by using H656/N746 and O777/N746. The fluctuations of PIR are quite small with the relative standard deviations close to $5 \%$. The calibration results for three kinds of fuel are nearly in the same fitted curve, indicating that the LIBS measurements are based on atoms and independent on molecular species.

It is known that the ambient pressure has significant effects on PIR in LIBS measurements, therefore, the calibration curves should be established at different ambient pressures. Figures 4(a) and (b) show the LIBS calibration curves at different ambient pressures. The 100 tests were conducted at each point, and the results in Fig. 4 are the averaged values. It can be seen that the PIRs correlate well with the atom concentration ratios at all three ambient pressures. In addition, the calibration curves keep consistent at different ambient pressures, indicating that the calibration results can be applied to the ambient pressures between 20 and 40 bar. This is critical since the injection process in engine cylinder involves the changing ambient pressure. The consistent calibration curves between 20 and 40 bar could be explained by the gas pressure effects on the PIR. Zhang et al. [22] reported that the line intensity ratio is significantly affected by the initial gas pressure lower than 2.0 bar, however, the effects become weaker and weaker with the increased gas pressure. At the gas pressure higher than 5.0 bar, the gas pressure effects on PIR are quite slight. The calibration results in Fig. 4 were employed in the concentration measurements in DI methanol-n-hexane blend sprays.

For the concentration measurements in high-pressure methanol-n-hexane blend sprays, the breakdowns are firstly produced at the targeted positions in sprays. After acquiring the emission spectra, the PIR of H656/N746 and 0777/N746 are determined. Combining with the calibration curves, the atom concentration ratios of $\mathrm{H} / \mathrm{N}$ and $\mathrm{O} / \mathrm{N}$ can be calculated. Since the oxygen atoms only originate from methanol and hydrogen atoms originate from both methanol and $\mathrm{n}$-hexane, the methanol concentration can be determined based on the atom concentration ratio of $\mathrm{O} / \mathrm{N}$. Then the n-hexane concentration can be determined based on the methanol concentration and the atom concentration ratio of $\mathrm{H} / \mathrm{N}$. As a result, the equivalence 

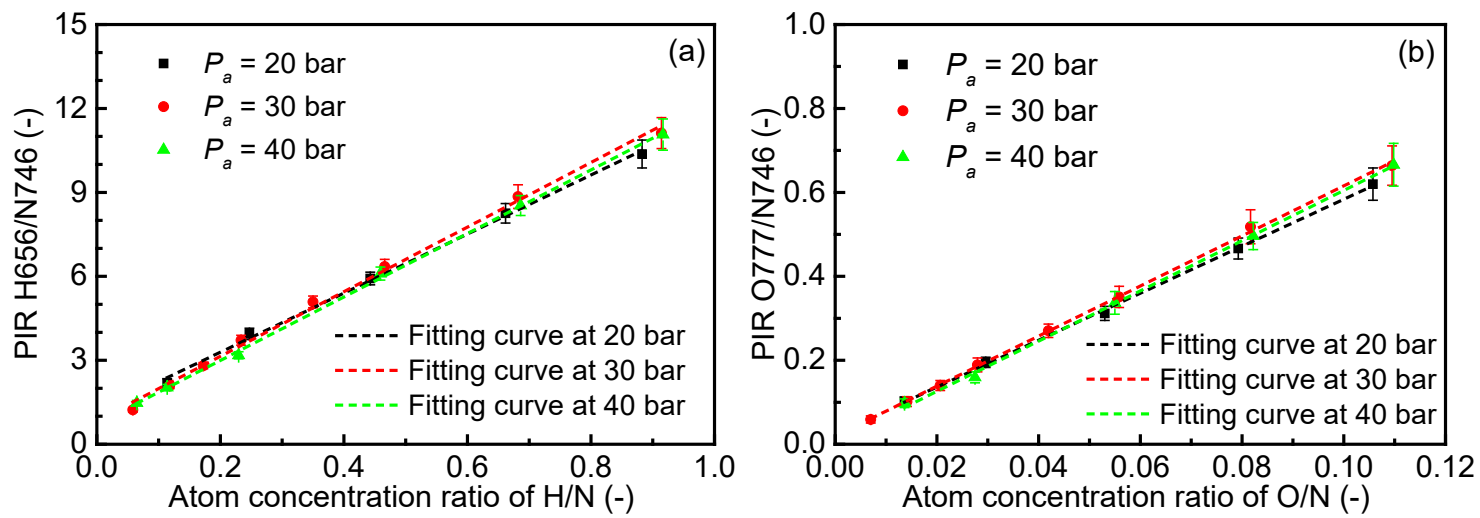

Figure 4. The LIBS calibration curves between (a) PIR of H656/N746 and atom concentration ratio of $H / N$ and (b) $\mathrm{PIR}$ of $\mathrm{O} 777 / \mathrm{N} 746$ and atom concentration ratio of $\mathrm{O} / \mathrm{N}$ at different ambient pressures $\left(E=137 \mathrm{~mJ}, T_{a}=460 \mathrm{~K}\right)$. ratio can be also estimated. The measurement uncertainties are estimated to be about $5 \%$ $10 \%$ by considering both the systematic errors including inaccuracies in calibrations and random errors including the pulse energy instability and the variations in laser-plasma interactions [13].

Figure 5(a) depicts the concentrations of methanol and n-hexane and the blending fraction in M15 spray at different radial distances from spray axis $(r)$. Ten tests were conducted at each measuring position. The results in Fig. 5(a) are the average values and the errors are the standard deviations of ten single tests. The blending fraction is defined as the mass fraction of methanol in the total fuel. It can be seen that both the n-hexane and methanol concentrations are highest at the jet axis and decrease with the radial distance increasing. As expected, remarkable variations of fuel concentration can be observed. Due to the different vaporization characteristics of individual components, M15 sprays would show a nonuniformed distribution of the blending fraction. For M15 fuel, the original mass fraction of methanol is $18 \%$ and the original mole fraction of methanol is $36 \%$. In general, the blending fraction is increased with the radial distance increasing except for the radial distance of 10 $\mathrm{mm}$. Due to the lower boiling temperature and higher vapor diffusivity of methanol, the methanol evaporates faster than n-hexane and therefore accumulates more at spray periphery region [23]. The result at the radial distance of $10 \mathrm{~mm}$ deviates from the trend as a result of the quite low concentrations of methanol and n-hexane.

Although nitrogen was employed as ambient gas, the effective equivalence ratio can be estimated by considering a $21 \% \mathrm{O}_{2}$ ambient. The calculation of effective equivalence ratio $(\phi)$ is based on the following reaction:

$$
x \cdot \mathrm{C}_{6} \mathrm{H}_{14}+y \cdot \mathrm{CH}_{4} \mathrm{O}+\frac{1}{\phi}\left(\frac{19 x+3 y}{2}\right) \cdot \mathrm{O}_{2}=(6 x+y) \cdot \mathrm{CO}_{2}+(7 x+2 y) \cdot \mathrm{H}_{2} \mathrm{O}
$$

where $x$ and $y$ are the mole fractions of $n$-hexane and methanol in the mixture, respectively. Then the equivalence ratio can be expressed as:

$$
\phi=\frac{19 x+3 y}{0.42(1-x-y)}
$$

The effective equivalence ratio of the mixture at different radial distances from spray axis is shown in Fig. 5(b). At the spray axis, the averaged equivalence ratio for ten tests is about 1.7, however, the single-shot results distribute in a wide range. The equivalence ratios in some laser shots are higher than 2.0, meaning the quite rich mixture. With the radial distance increasing, the equivalence ratio significantly reduces due to the air entrainment caused by 


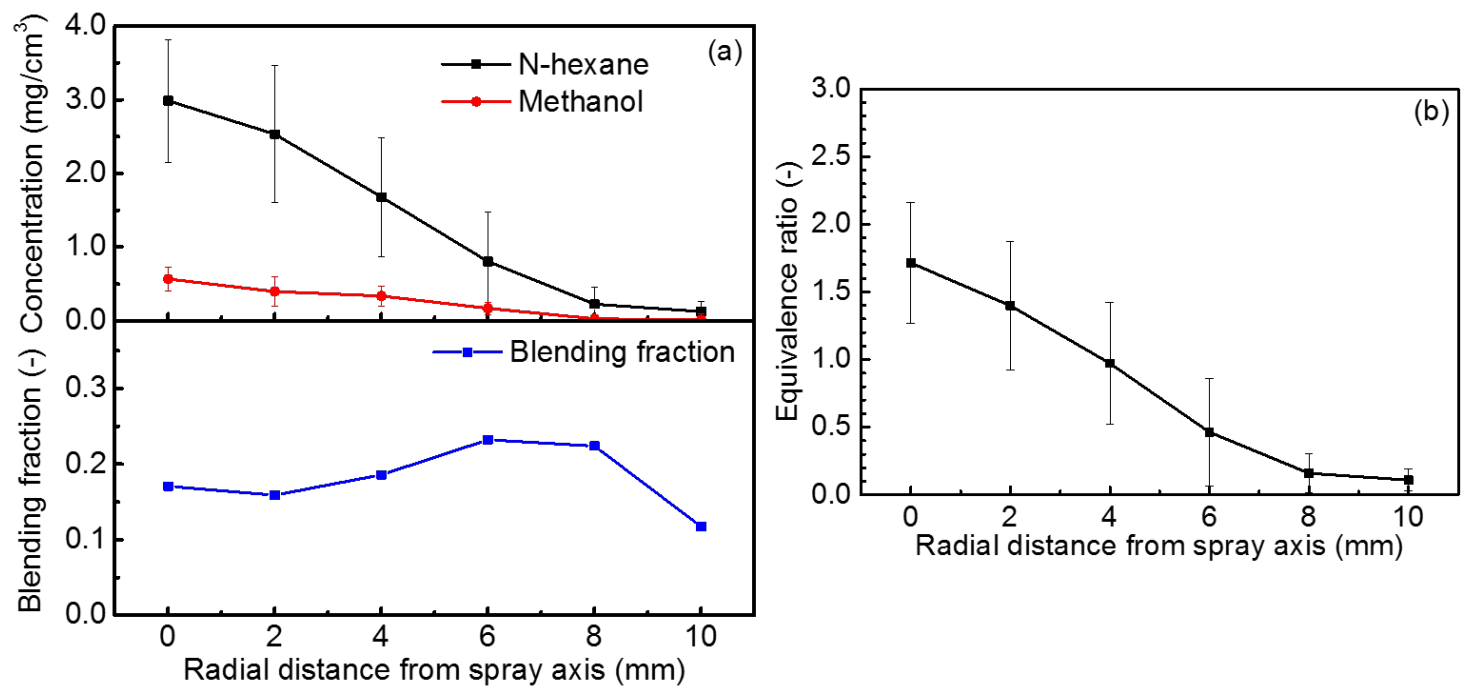

Figure 5. (a) The concentrations of methanol and n-hexane and the blending fraction in M15 spray at different radial distances from spray axis, (b) radial distribution of equivalence ratio ( $P_{i n j}=60 \mathrm{Mpa}, P_{a}=20 \mathrm{bar}, T_{a}=460 \mathrm{~K}$, $z=40 \mathrm{~mm})$.

the strong turbulence at the spray edges. The equivalence ratio at $4 \mathrm{~mm}$ from spray axis is about 1.0 , indicating a suitable ignition position considering the fuel concentration.

\section{Conclusions}

A novel approach based on LIBS has been proposed to quantitatively and simultaneously measure the fuel concentrations of methanol and $n$-hexane by using the multiple atomic lines. The LIBS calibration results shows that for three kinds of fuels, the PIRs of H656/N746 and O777/N746 linearly correlate well with the atom concentration ratios of $\mathrm{H} / \mathrm{N}$ and $\mathrm{O} / \mathrm{N}$, respectively, indicating the potentials of measurements of multi-component fuels. In addition, the calibration curves keep consistent at different ambient pressures, making the approach applicable in real engines, i.e., varied pressure during the injection period. M15 spray shows a non-uniformed radial distribution of the blending fraction. The blending fraction is generally increased with the radial distance increasing because of the lower boiling temperature and higher vapor diffusivity of methanol than $\mathrm{n}$-hexane.

\section{Acknowledgments}

The study is supported by the National Natural Science Foundation of China (51776125).

\section{References}

[1] Liu, S., Cuty Clemente, E. R., Hu, T., and Wei, Y., 2007, Applied Thermal Engineering, 27(11-12), pp. 19041910.

[2] Agarwal, A. K., Karare, H., and Dhar, A., 2014, Fuel Processing Technology, 121, pp. 16-24.

[3] Stan, C., Tröger, R., and Stanciu, A., 2000, SAE paper 2000-01-2904.

[4] Williams, B., Edwards, M., Stone, R., Williams, J., and Ewart, P., 2014, Combustion and Flame, 161(1), pp. 270-279.

[5] Szybist, J., Foster, M., Moore, W. R., Confer, K., Youngquist, A., and Wagner, R., 2010, SAE paper 2010-010619.

[6] Mohd Murad, S. H., Camm, J., Davy, M., Stone, R., and Richardson, D., 2016, SAE paper 2016-01-0991.

[7] Samimi Abianeh, O., and Chen, C. P., 2012, International Journal of Heat and Mass Transfer, 55(23), pp. 68976907.

[8] Stagni, A., Esclapez, L., Govindaraju, P., Cuoci, A., Faravelli, T., and Ihme, M., 2017, Proceedings of the Combustion Institute, 36(2), pp. 2483-2491.

[9] Zhang, Z., Li, T., and Shi, W., 2019, Energy, 171, pp. 372-384.

[10] Ben, L., Charnay, G., Bazile, R., and Ferret, B., 2007, Experiments in Fluids, 43(1), pp. 77-88.

[11] Li, T., Nishida, K., and Hiroyasu, H., 2011, Fuel, 90(7), pp. 2367-2376.

[12] Li, S., Zhang, Y., and Qi, W., 2018, Experimental Thermal and Fluid Science, 98, pp. 472-479.

[13] Zhang, Z., Li, T., Xue, X., and Huang, S., 2019, Spectrochimica Acta Part B: Atomic Spectroscopy, 161, p. 105706.

[14] Lee, S. H., Do, H., and Yoh, J. J., 2016, Combustion and Flame, 165, pp. 334-345. 
[15] Schulz, C., and Sick, V., 2005, Progress in Energy \& Combustion Science, 31(1), pp. 75-121.

[16] Zeng, W., Xu, M., Zhang, M., Zhang, Y., and Cleary, D., 2010, SAE paper 2010-01-0602.

[17] Andersson, M., Warnberg, J., Hemdal, S., Dahlander, P., and Denbratt, I., 2011, SAE paper 2011-01-1889.

[18] Li, T., Hiroyasu, H., Zhang, Y., and Nishida, K., 2003, SAE paper 2003-01-3161.

[19] Hunter, A. J. R., Holtzclaw, K. W., Piper, L. G., Fraser, M. E., and Davis, S. J., 2000, Applied Spectroscopy, 54(4), pp. 575-582.

[20] Kawahara, N., Tsuboi, K., and Tomita, E., 2014, Optics Express, 22(S1), pp. A44-A52.

[21] Abdul Rahman, M. T., Kawahara, N., Tsuboi, K., and Tomita, E., 2014, International Journal of Hydrogen Energy, 39(31), pp. 17896-17905.

[22] Zhang, Z., Li, T., and Huang, S., 2019, Spectrochimica Acta Part B: Atomic Spectroscopy, 155, pp. 24-33.

[23] Chen, R., Nishida, K., and Shi, B., 2019, Fuel, 242, pp. 425-437.

[24] Wei, Y., Li, T., Zhou, X., and Zhang, Z., 2020, Fuel, 268, p. 117343. 